INRA Prod. Anim.,

2014, 27 (2), 135-146

\title{
Conception d'un système innovant de production de truites en eau recirculée
}

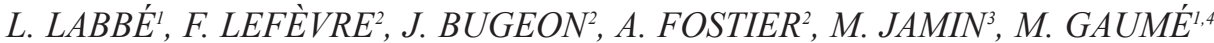 \\ ${ }^{I}$ INRA, UE937, PEIMA, F-29450 Sizun, France \\ 2 INRA, UR1037, LPGP, F-35042 Campus de Beaulieu, Rennes, France \\ 3 Fili@vet, cabinet vétérinaire aquacole, 15 rue du puits, F-29600 Saint Martin des Champs, France \\ ${ }^{4}$ ITAVI Section aquacole F-75008 Paris, France \\ Courriel :Laurent.Labbe@rennes.inra.fr
}

\begin{abstract}
L'élevage des poissons est fortement tributaire du milieu naturel. En effet, l'eau utilisée comme milieu d'élevage y est prélevée, puis restituée. L'élevage de truites en eau recirculée, susceptible de réduire de $90 \%$ les besoins en eau d'une salmoniculture, est une piste d'avenir pour une aquaculture moins dépendante de son environnement.
\end{abstract}

L'aquaculture est le secteur agroalimentaire qui connaît la plus forte croissance mondiale. En 2010, sur 128 millions de tonnes de poissons destinés à la consommation humaine, produits par les captures de pêche et l'aquaculture, $47 \%$ proviennent de cette dernière (FAO 2011). Pendant la même période la production française de poissons d'élevage s'est élevée à 50000 tonnes ce qui ne représente que $3 \%$ de la consommation totale (FranceAgriMer 2011). Les causes du faible développement de la pisciculture en France sont diverses et relativement complexes. La mission sur le développement de l'aquaculture menée en 2008 par la députée Hélène Tanguy (Tanguy 2008) révèle qu'une des principales contraintes au développement de cette filière est d'ordre environnemental et porte notamment sur l'usage de l'eau et le respect de normes de rejet dans l'effluent. Par rapport aux autres activités d'élevage, les relations entre pisciculture et milieu sont particulièrement étroites. Dans un système d'élevage de poissons classique en Circuit Ouvert (CO), l'eau utilisée est prélevée dans le milieu naturel aquatique, traverse les bassins d'élevage et est restituée. La quantité d'eau nécessaire comme milieu d'élevage pour produire un kilogramme de truite en circuit traditionnel ouvert est de l'ordre $100 \mathrm{~m}^{3}$. Cette quantité d'eau disponible, notamment en période d'étiage, conditionne la capacité de production de l'ensemble du site sur une année. En effet le cycle de production dure de 10 à 12 mois pour une truite portion $(250 \mathrm{~g})$ et de 20 à 22 mois pour une grande truite $(2,5 \mathrm{~kg})$. Par ailleurs, l'utilisation d'une partie de l'eau des rivières peut également poser des problèmes pour la libre circulation des sal- monidés migrateurs (saumons, truites fario...) à certaines périodes. Enfin, la salmoniculture est soumise à de fortes contraintes environnementales encadrées par des directives européennes (DCE 2000, EMA 2006). Afin de proposer une solution technique permettant d'optimiser et d'augmenter le potentiel des sites installés sur des rivières au débit moyen, et de s'affranchir des conditions pluviométriques et des facteurs abiotiques tout en diminuant l'impact environnemental, la Pisciculture Expérimentale Inra des Monts d'Arrée (PEIMA) a mis au point et éprouvé un système d'élevage des salmonidés basé sur la recirculation de l'eau.

\section{1 / La pisciculture en eau recirculée}

L'élevage piscicole en Circuit Recirculé (CR) désigne un éventail de techniques dont le but est de restreindre et de maîtriser les interactions entre les poissons d'élevage et l'écosystème aquatique. Cette technique repose sur une combinaison de processus biologiques contrôlant les rejets cataboliques toxiques (transformation de l'ammoniaque en nitrite puis nitrate), de techniques d'élimination des particules solides (filtration mécanique, décantation), et de contrôle des gaz dissous (apports en oxygène, dégazage du $\mathrm{CO}_{2}$ ). L'élevage en eau recirculée est utilisé depuis la fin des années 1980 pour la production de différentes espèces de poissons généralement à haute valeur ajoutée. Ce type de système d'élevage s'est d'abord développé en eau douce pour des poissons nécessitant des eaux chaudes (poisson chat africain, tilapia), mais la compétition avec les importations de pangasius
d'Asie va remettre en cause la rentabilité de ces productions (Van Duijn et al 2010). Il s'est ensuite développé en eau de mer pour la production de turbot et de juvéniles de bar ou de daurade (Blancheton et al 2009). L'utilisation de circuit d'eau recirculée pour le pré-grossissement suivi d'un grossissement en $\mathrm{CO}$ se développe aux Iles Féroé et en Norvège pour la production de smolts de saumon Atlantique (Kristen et al 2009). Le contrôle de la qualité d'eau sur ces stades plus fragiles permet de raccourcir le cycle d'élevage, de s'affranchir des variations saisonnières de débit et de température et aussi, d'obtenir des animaux mieux adaptés aux phases de grossissement.

Plus récemment, de tels systèmes ont été développés pour des élevages de salmonidés en eau douce ou en eau de mer au Danemark et en France, afin de faire face à une pression environnementale forte. Roque d'Orbcastel et al (2009b) ont comparé le bilan environnemental d'un Système en eau Recirculé (SR) de production de truites en eau douce avec celui de deux Systèmes Ouverts (SO) par la méthode de l'Analyse du Cycle de Vie (ACV). Cette analyse a démontré que « le bilan environnemental du SR est plus avantageux que celui du SO à la fois au niveau global, avec un potentiel de réchauffement climatique moindre et moins d'utilisation de production primaire, et au niveau régional avec une forte réduction des consommations en eau, de l'espace utilisé et des impacts potentiels sur l'eutrophisation et l'acidification du milieu ». En dépit de ces avantages environnementaux, la part de la production issue de systèmes en eau recirculée reste faible en Europe (Martins et al 2010) et surtout en France. Les 
principales raisons évoquées par les producteurs sont la lourdeur des investissements liés à la construction de nouveaux sites (Schneider et al 2006), le coût énergétique de la recirculation de l'eau et de son traitement, évalué entre 3 et $6 \mathrm{kwh} . \mathrm{kg}^{-1}$ de poisson produit, l'apparition de défauts de flaveurs (Roque d'Orbcastel et al 2008) ainsi que la difficulté à maîtriser un outil qui peut paraître techniquement plus complexe à gérer qu'un élevage traditionnel.

L'étude conduite à la PEIMA avait pour objectif de valider les solutions techniques permettant de lever une partie de ces contraintes. Nous avons d'abord vérifié si nous pouvions transformer un système de production ouvert, alimenté en eau de rivière en SR tout en mettant en œuvre des techniques et des outils de traitement de l'eau couramment utilisés en SO intensif (plateforme à jet, pompes de recyclages et filtre à tambour). Puis nous avons défini les limites du système en termes d'apport d'eau neuve, exprimé en $\mathrm{m}^{3} \cdot \mathrm{kg}^{-1} \mathrm{~d}$ 'aliment distribué par jour, et de taux de recirculation de l'eau, exprimé en taux de renouvellement par heure dans les bacs d'élevage. L'apport d'eau neuve a été fixé à $7 \mathrm{~m}^{3} \cdot \mathrm{kg}^{-1}$ d'aliment. Cet apport dépend du degré de sophistication de la boucle de traitement de l'eau et de son efficacité. C'est cette eau qui va permettre l'évacuation des déchets non traités (Matières En Suspension (MES) fines non collectées, nitrates, $\mathrm{CO}_{2}$ résiduel) et ainsi de maintenir une qualité d'eau et une température compatibles avec l'élevage. Le taux de renouvellement d'eau dans les bacs a été fixé à 2,5 par heure. Ce taux offre un compromis intéressant entre la consommation énergétique et le maintien d'une bonne qualité d'eau. En deçà, l'évacuation du $\mathrm{CO}_{2}$ produit par les poissons n'est plus assurée et nous avons vu apparaître des phénomènes d'hypercapnie incompatibles avec le bon état sanitaire des animaux et leur croissance. Au-delà, il génère une consommation énergétique supplémentaire sans amélioration notable de la qualité d'eau de l'élevage ni des performances de croissance.

Après avoir validé les options techniques choisies, nous avons comparé ce système d'élevage avec un système d'élevage traditionnel en $\mathrm{CO}$ situé au même endroit. L'évaluation de l'effet de la réduction de la quantité d'eau utilisée a porté sur i) les paramètres physicochimiques de la qualité d'eau, ii) la croissance des poissons et les principaux indicateurs zootechniques (survie, indice de consommation), iii) la pathologie et notamment le parasitisme, iv) la reproduction des poissons (taux de maturation, fécondité, taux de fécondation, taux de survie au stade embryonné) v) la qualité des carcasses et de la chair (ren-
Figure 1. Schéma simplifié du système recirculé.

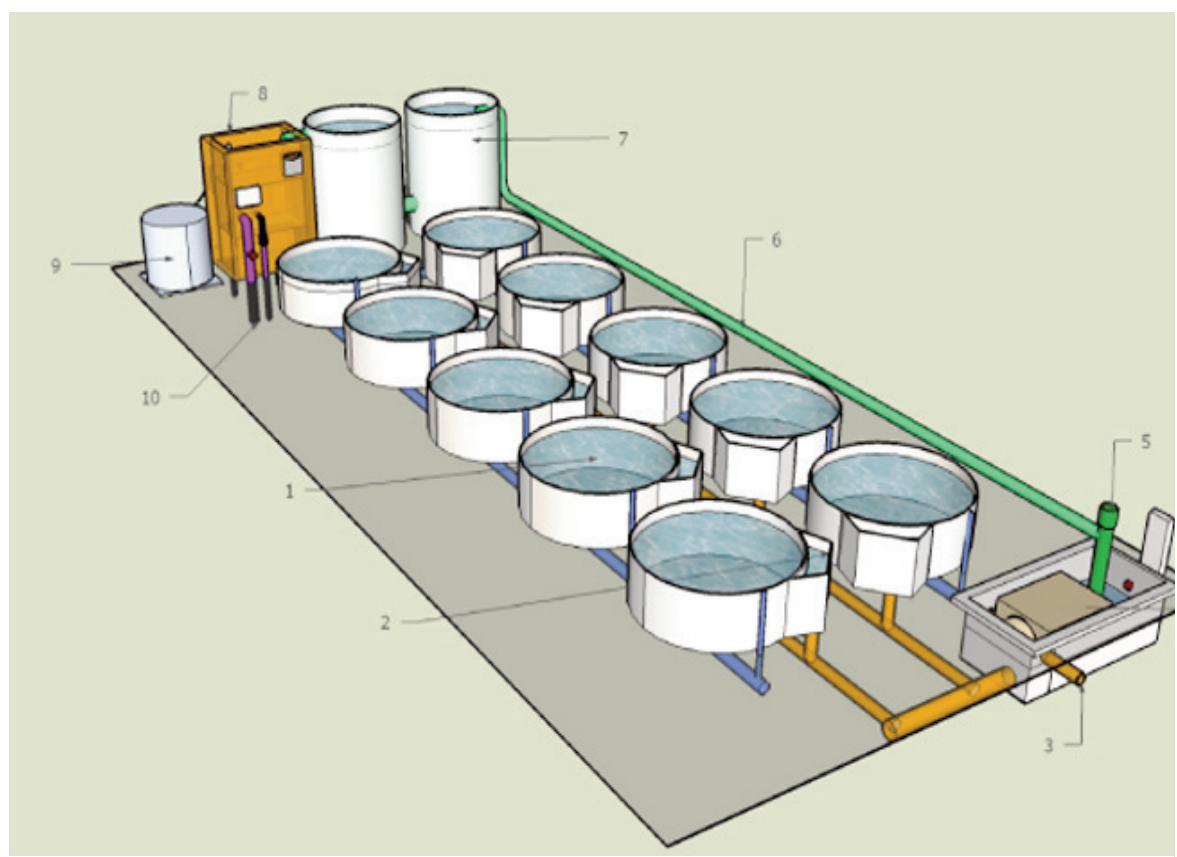

1) Bacs d'élevage (6 $\mathrm{m}^{3}$ utiles) ; 2) Décanteurs externes ; 3) Evacuation du trop plein et du contre lavage du filtre rotatif ; 4) Filtre à tambour $(65 \mu)$; 5) Pompe (max $\left.140 \mathrm{~m}^{3} \cdot \mathrm{h}^{-1}\right)$; 6) injection de bicarbonate de sodium ; 7) Cuves contenant le filtre biologique $\left(2 \times 6,7 \mathrm{~m}^{3}\right.$ en série $\left.) ; 8\right)$ Plate-forme à jet (système d'injection d' $\left.\mathrm{O}_{2}\right)$; $9)$ Réservoir d'oxygène ; 10) Entrée d'eau neuve. dement au filetage, qualité physico chimique et qualité organoleptique du filet).

\section{2 / Le pilote développé à la PEIMA}

\section{1 / Le système recirculé}

Dans la suite de cet article, les caractéristiques du système et les caractères mesurés sur les animaux seront indexés selon les identifiants utilisés dans les ontologies EOL et ATOL (http://www.atolontology.com/, Golik 2012).
Le pilote ( figure 1), c'est-à-dire l'ensemble d'équipements ou de dispositifs permettant de tester un procédé à une échelle ou dans un environnement proche de la réalité industrielle, a été réalisé en transformant une plateforme qui fonctionnait selon un schéma de production classique en CO (EOL:0001571) en système de production en CR (EOL: 0001508). Cette plateforme compte 10 bassins circulaires (EOL:0001532) en résine de polyester de $3 \mathrm{~m}$ de diamètre. Elle a été équipée d'une pompe de relevage et d'un filtre biologique. Le filtre à tambour et le système d'oxygénation

\section{Encadré. Le filtre biologique.}

Le filtre biologique (figure 2) est l'élément clé de ce système d'élevage. II est ici composé de deux cuves cylindriques en résine de $6,7 \mathrm{~m}^{3}$ chacune installée en série. Les deux réservoirs sont remplis de $3,2 \mathrm{~m}^{3}$ d'anneaux en polypropylène dont la surface spécifique est de $800 \mathrm{~m}^{2}$ par $\mathrm{m}^{3}$. Ces anneaux servent de support aux bactéries et sont mis en mouvement par une injection d'air pulsé. Le filtre biologique à lit fluidisé a trois avantages : i) il ne nécessite que peu d'entretien, ii) sa conception permet d'optimiser la surface de contact entre les bactéries et le substrat et enfin iii) il permet l'évacuation du $\mathrm{CO}_{2}$ et un apport d' $\mathrm{O}_{2}$ nécessaire à la dégradation bactérienne du $\mathrm{NH}_{4}{ }^{+}$. Son bon fonctionnement est garant de la qualité de l'eau du système. La filtration biologique réduit les teneurs en ammoniaque de l'eau en transformant le couple $\mathrm{NH}_{3} / \mathrm{NH}_{4}{ }^{+}$en nitrites puis nitrates, dont les concentrations tolérées par les poissons sont largement supérieures. Pour les truites, la croissance n'est affectée que pour des concentrations supérieures à $180 \mathrm{mg} \cdot \mathrm{L}^{1}$ de N-NO $3^{-}$(Berka et al 1981). Le filtre biologique a donc pour but d'offrir des conditions optimales au développement des bactéries autotrophes nitrifiantes, de la famille des Nitrosomonas et des Nitrobacter qui agissent en deux étapes successives selon les équations suivantes

$$
\begin{array}{ll}
2 \mathrm{NH}_{4}^{+}+3 \mathrm{O}_{2} \text { à } 2 \mathrm{NO}_{2}^{-}+4 \mathrm{H}^{+}+2 \mathrm{H}_{2} \mathrm{O} & \text { (Nitrosomonas) } \\
2 \mathrm{NO}_{2}^{-}+\mathrm{O}_{2} \text { à } 2 \mathrm{NO}_{3^{-}} & \text {(Nitrobacter) }
\end{array}
$$

Ces réactions provoquent une acidification du milieu par libération d'ions $\mathrm{H}^{+}$. Pour compenser cette acidification et contrôler le $\mathrm{pH}$ nous ajoutons du bicarbonate de sodium à hauteur d'un quart de la quantité d'aliment distribuée (masse à masse). 
Figure 2. Vue tridimensionnelle en transparence des deux cuves composant le filtre biologique.
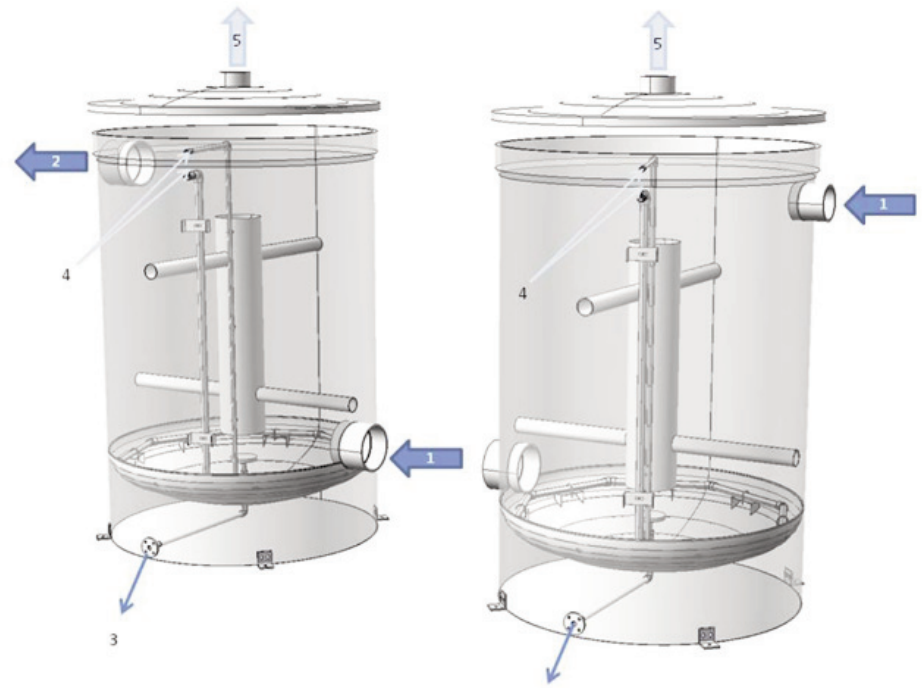

1) Alimentation en eau brute $\left.\left(140 m^{3} \cdot h^{-1}\right) ; 2\right)$ Evacuation de l'eau traitée ; 3) Vanne de vidange ; 4) Injection d'air (70 $\mathrm{m}^{3} \cdot \mathrm{h}^{-1}$ par filtre) ; 5) Evacuation de l'air.

étaient déjà présents. L'eau issue des bacs d'élevage est débarrassée des MES grâce à un filtre mécanique d'une maille de $63 \mu \mathrm{m}$. L'eau recirculée est envoyée sur le filtre biologique (cf. encadré et figure 2) à l'aide d'une pompe élévatrice.

La dernière étape du traitement de l'eau dans ce pilote est la ré-oxygénation. La plateforme à jet est l'élément du circuit permettant le maintien d'une concentration en $\mathrm{O}_{2}$ dissous optimale pour satisfaire les besoins des poissons. Après ré-oxygénation l'eau alimente de nouveau les bassins d'élevage où elle apporte aux poissons l'oxygène nécessaire et évacue les déchets qu'ils ont produits $\left(\mathrm{CO}_{2}\right.$, $\mathrm{NH}_{4}{ }^{+}$et MES).

\section{2 / Le système ouvert}

Le CO utilisé dans cette étude pour comparer les performances des truites dans les deux systèmes est composé d'un groupe de 6 bassins circulaires de $2 \mathrm{~m}$ de diamètre et d'un volume utile de $1,8 \mathrm{~m}^{3}$. Chaque bassin est alimenté en « eau neuve » par deux tuyaux différents. Le premier fournit le flux principal d'eau du système. Le second est le système d'adduction d'eau suroxygénée. Cette eau suroxygénée provient d'un système d'oxygénation à double cône alimenté à $1^{\prime} \mathrm{O}_{2}$ pur. Ce système permet d'atteindre des niveaux de sursaturation dans l'eau d'élevage, équivalents à ceux pratiqués dans le SR.

\section{3 / Qualité de l'eau}

\section{1 / Prélèvements et mesures}

Les mesures de qualité d'eau du CR ont été effectuées pendant la durée com- plète d'un cycle d'élevage de grandes truites (610 jours). L'objectif de ces mesures est de vérifier que le choix des ouvrages d'épuration de l'eau et leur dimensionnement permettent de maintenir une qualité d'eau compatible avec la santé, le bien-être et la croissance des animaux. Enfin, nous avons cherché à modéliser la production des effluents de ce système. Six bilans complets sur un cycle de $24 \mathrm{~h}$ (1 prélèvement toutes les heures) ont été réalisés entre mars 2011 et novembre 2012. Ils correspondent à des stades d'élevages et des températures différentes $\left(8,11,12,13,14\right.$ et $\left.16^{\circ} \mathrm{C}\right)$. La durée d'échantillonnage a été fixée à $24 \mathrm{~h}$ pour correspondre au cycle de la distribution de l'aliment et d'excrétion des effluents. L'azote ammoniacal $\left(\mathrm{N}-\mathrm{NH}_{4}{ }^{+}\right.$,

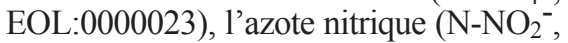
EOL:0000198), l'azote nitreux (N-NO${ }_{3}^{-}$,' EOL:0000024) les ortho-phosphates $\left(\mathrm{P}-\mathrm{PO}_{4}{ }^{3-}, \mathrm{EOL}: 0001488\right)$ ont été mesurés par spectrométrie. Pour éviter tout biais dans la prise de mesure, des échantillons ont été collectés à la main sur cinq points stratégiques du circuit : $i$ ) entrée du filtre biologique, ii) sortie du filtre biologique, iii) eau neuve, $i v$ ) sortie des bacs d'élevage et $v$ ) contre-lavage du filtre rotatif. En parallèle la concentration de l'eau en $\mathrm{O}_{2}$ (EOL:0001481) en entrée et sortie de plateforme à jet a été enregistrée. La concentration en $\mathrm{CO}_{2}$ (EOL:0000263) a été enregistrée en sortie de filtre biologique et en sortie de bassin. Les échantillons pour les MES ont été réalisés selon la Norme Européenne NF EN 8726. Les boues collectées dans les décanteurs ont été déshydratées à la PEIMA (étuve à $105^{\circ} \mathrm{C}$ ) puis analysées par le laboratoire CAPINOV. Lors de ces mesures, le taux d'ouverture du circuit est resté constant à $7,2 \pm 0,8 \mathrm{~m}^{3} \cdot \mathrm{h}^{-1}$. La quantité quotidienne d'aliment distribuée a été de $21 \pm 1,5 \mathrm{~kg}$.
La biomasse présente dans le circuit était de $1965 \pm 374 \mathrm{~kg}$. Les poissons ont été nourris deux fois par jour avec deux types d'aliment commercial Biomar ${ }^{\circledR}$ de la gamme Efico YS (871 et 691); l'aliment 871 avait une teneur en protéines de $40 \%$ (EOL:0000124) et en lipides de 30\% (EOL:0000082), l'aliment 691 de $43 \%$ en protéines et de $24 \%$ en lipides. Le taux de rationnement (EOL:0001748) a été calculé en fonction du poids moyen des poissons et de la température de l'eau (EOL:0000036). Il a été réactualisé toutes les semaines grâce au logiciel de gestion zootechnique ECU 2000 développé à la PEIMA basé sur le modèle de croissance de Muller Feuga et sur un modèle d'indice de consommation (Fauré et Labbé 1999).

\section{2 / L'eau entrante ou « eau neuve "}

L'eau d'alimentation de la pisciculture provient du lac du Drennec. Elle est captée de manière gravitaire depuis le fond du lac puis passe à travers un dégazeur et un filtre mécanique à tambour à $100 \mu \mathrm{m}$ avant d'être répartie entre les différentes structures d'élevage. La qualité physico-chimique de l'eau n'est pas stable au cours de l'année. Du fait du dégazage, la teneur en $\mathrm{O}_{2}$ est toujours proche de la saturation et le $\mathrm{CO}_{2}$ est présent à des teneurs inférieures à $3 \mathrm{mg} . \mathrm{L}^{-1}$. D'une manière générale l'eau est acide $(5,9<\mathrm{pH}<7$, EOL:0000015) et faiblement minéralisée. La température moyenne (EOL:0000247) sur la période est de $12,3^{\circ} \mathrm{C}$ degré avec un minimum à $5^{\circ} \mathrm{C}$ (EOL:0000246) et un maximum à $18,6^{\circ} \mathrm{C}(\mathrm{EOL}: 0000176)$. Les teneurs exprimées en mg.L-1 pour les paramètres suivants ont été de : $\mathrm{N}_{-} \mathrm{NH}_{4}{ }^{+}: 0,127 \pm 0,1$; $\mathrm{N}-\mathrm{NO}_{2}{ }^{-}: 0,013 \pm 0,007, \mathrm{~N}-\mathrm{NO}_{3}: 1,46 \pm$ 0,66. Les teneurs en MES (EOL:0000027) ont été de $0,8 \pm 0,3 \mathrm{mg} . \mathrm{L}^{-1}$. Cette eau sera nommée dans le reste de cet article « l'eau neuve ».

\section{3 / L'eau des bassins d'élevage}

La qualité de l'eau dans les bassins d'élevage du CR est détaillée dans le tableau 1. Les mesures et analyses ont montré que la concentration en MES, $\mathrm{N}-\mathrm{NO}_{2}{ }^{-}, \mathrm{N}-\mathrm{NO}_{3}^{-}, \mathrm{N}_{-} \mathrm{NH}_{4}{ }^{+}$ont toujours été inférieures aux valeurs seuils requises pour l'élevage des salmonidés (tableau 1) (Roque d'Orbcastel 2008). La concentration en $\mathrm{O}_{2}$ en sortie a toujours été supérieure à $5,5 \mathrm{mg} . \mathrm{L}^{-1}$. La concentration moyenne en $\mathrm{CO}_{2}$ a été de $13 \pm 5,1 \mathrm{mg}$. $\mathrm{L}^{-1}$. La température moyenne sur la période a été de $13,1^{\circ} \mathrm{C}$ ( soit $0,6^{\circ} \mathrm{C}$ de plus que l'eau neuve sur la même période. L'amplitude de température a été de $16^{\circ} \mathrm{C}$ (EOL: 0000035) pour l'eau du CR avec un minimum à $4,5^{\circ} \mathrm{C}$ et un maximum à $20,5^{\circ} \mathrm{C}$ alors que celle du CO n'a été que de $13,6^{\circ} \mathrm{C}$ avec un minimum à $5^{\circ} \mathrm{C}$ et un maximum à $18,6^{\circ} \mathrm{C}$. 
Tableau 1. Qualité de l'eau dans le circuit recirculé (CR) et valeurs seuils pour l'élevage en eau douce des salmonidés.

\begin{tabular}{|c|c|c|c|c|c|c|c|c|}
\hline & pH & $\begin{array}{c}\mathbf{T} \\
\left({ }^{\circ} \mathrm{C}\right)\end{array}$ & $\begin{array}{c}\mathbf{O}_{\mathbf{2}} \\
\left(\mathrm{mg} \cdot \mathrm{L}^{-1}\right)\end{array}$ & $\begin{array}{c}\mathbf{C O}_{2} \\
\left(\mathrm{mg} \cdot \mathrm{L}^{-1}\right)\end{array}$ & $\begin{array}{c}\text { MES } \\
\left(\mathrm{mg}^{-1} \mathrm{~L}^{-1}\right)\end{array}$ & $\begin{array}{r}\mathbf{N}-\mathbf{N H}_{4}{ }^{+} \\
\left(\mathrm{mg}^{-1} \mathrm{~L}^{-1}\right)\end{array}$ & $\begin{array}{l}\mathrm{N}-\mathrm{NO}_{2-} \\
\left(\mathrm{mg} \cdot \mathrm{L}^{-1}\right)\end{array}$ & $\begin{array}{c}\mathrm{N}-\mathrm{NO}_{3}- \\
\left(\mathrm{mg} \cdot \mathrm{L}^{-1}\right)\end{array}$ \\
\hline Moyenne & 6,9 & 13,1 & 8 & 12,6 & 2,9 & 0,66 & 0,12 & 5,46 \\
\hline Maximum & 7,3 & 20,5 & 14 & 18,0 & 3,7 & 0,93 & 0,22 & 6,47 \\
\hline Minimum & 6,4 & 4,5 & 5,5 & 10,2 & 2,3 & 0,44 & 0,08 & 4,51 \\
\hline Valeurs seuil & $6-8$ & $<25$ & $>6-7$ & $<15-20$ & $<80$ & $<1-1,5$ & $<0,2-0,3$ & $<180$ \\
\hline
\end{tabular}

\section{4 / Efficacité du système de traitement de l'eau du CR}

Le débit d'air fourni par le compresseur a été de $140 \mathrm{~m}^{3} \cdot \mathrm{h}^{-1}$. Les rendements d'oxygénation et de dégazage du $\mathrm{CO}_{2}$ de l'air lift des filtres biologiques ont été calculés par des bilans de matières $\left(\mathrm{O}_{2}\right.$ et $\left.\mathrm{CO}_{2}\right)$ en entrée et sortie du filtre biologique $(n=3)$. Ils sont de $16 \pm 10,1 \%$ pour l' $\mathrm{O}_{2}$ et de $46 \pm 2 \%$ pour le $\mathrm{CO}_{2}$. Le rendement d'oxygénation de la plateforme à jet qui est fonction du taux de saturation désiré en sortie a varié de 83 à $95,8 \%$. Le rendement du filtre biologique, c'est-à-dire sa capacité à abattre l'azote ammoniacal, a été calculé sur la base de prélèvements d'eau en entrée et sortie de ce filtre. Six bilans de $24 \mathrm{~h}$ ont été réalisés, soit 288 mesures. Le rendement moyen sur la période est de $33,7 \pm 4,1 \%$. Le filtre biologique a fonctionné avec $21 \mathrm{~m}^{3}$ d'air.h-1 $\mathrm{h}^{-1}$ de substrat. La cinétique d'oxydation de $\mathrm{N}-\mathrm{NH}_{4}$ par le filtre biologique a été en moyenne sur la période de 610 jours de $67,5 \pm 8,5 \mathrm{~g}$ de $\mathrm{N}-\mathrm{NH}_{4}{ }^{+} \cdot \mathrm{m}^{-3}$ de substrat.j-1 pour une concentration de $\mathrm{N}-\mathrm{NH}_{4}{ }^{+}$en entrée de filtre de $0,57 \pm 0,18 \mathrm{mg} . \mathrm{L}^{-1}$ et une concentration résiduelle de $\mathrm{N}-\mathrm{NO}_{2}-$ de $0,12 \pm 0,05 \mathrm{mg} \cdot \mathrm{L}^{-1}$. L'efficacité de traitement des rejets solides par les décanteurs a été évaluée à $39,5 \% \pm 5 \%$ alors que le filtre rotatif en a capté $60 \% \pm 5 \%$.

\section{5 / Evaluation des rejets du CR}

Le traitement et la valorisation des rejets issus d'un CR ne peuvent être définis sans la connaissance et la compréhension des transferts et transformations de matières introduites au sein du système. Nous avons essayé de documenter et de modéliser son fonctionnement à travers un bilan de masse des différentes fractions de l'azote et des MES à l'intérieur du circuit et exportées. Les valeurs obtenues par méthode hydrobiologique ont été comparées aux valeurs théoriques obtenues par le modèle nutritionnel développé par Papatryphon et al (2005). La méthode hydro-biologique consiste à procéder à des prises d'échantillons d'eau en différents points du circuit et des mesures des débits d'eau neuve, d'eau recirculée pour chaque bassin d'élevage. Des bilans de masse sont réalisés sur les composés azotés, solides et dissous, collectés selon les méthodes et aux dates décrites précédem- ment. Pour les MES, la valeur mesurée est de $131 \pm 12 \mathrm{~g}$ de matière sèche par kg d'aliment distribué. Le résultat estimé par le calcul est de $133 \pm 13 \mathrm{~g}$ par $\mathrm{kg}$ d'aliment. En ce qui concerne le $\mathrm{N}-\mathrm{NH}_{4}{ }^{+}$, la valeur mesurée de $25,1 \pm 1,8 \mathrm{~g}$ par $\mathrm{kg}$ d'aliment est significativement $(\mathrm{P}=0,013)$ inférieure à la valeur prédite $(27,7 \pm 2,7 \mathrm{~g})$. La modélisation de la quantité de phosphore excrétée par les poissons est plus complexe. Les résultats obtenus lors des quatre premières campagnes de mesure $\left(\mathrm{P}-\mathrm{PO}_{4}{ }^{(3-)}\right)$ ne sont malheureusement pas exploitables du fait de résultats trop variables probablement dus au protocole d'analyse utilisé. Seuls les résultats des $5^{\text {e }}$ et $6^{\text {e }}$ campagnes basés sur le $P$ total mesuré en différents points du circuit et dans les fèces collectées dans les décanteurs ont pu être utilisés. La quantité prédite par le calcul est de 4,48 $\pm 0,01 \mathrm{~g}$ de $\mathrm{P}$ total par $\mathrm{kg}$ d'aliment. Nous avons mesuré une production de $6,45 \pm 0,5 \mathrm{~g}$ par kg d'aliment.

\section{6 / Discussion}

Dans le système d'élevage en CR, la qualité de l'eau est restée conforme aux recommandations pour l'élevage des salmonidés (cf. tableau 1). La concentration en $\mathrm{O}_{2}$ en sortie de bassin a toujours été supérieure à $5,5 \mathrm{mg}$. $\mathrm{L}^{-1}$ et les concentrations en $\mathrm{N}_{-} \mathrm{NH}_{4}{ }^{+}$et $\mathrm{N}_{-} \mathrm{NO}_{2}{ }^{-}$ sont restées inférieures à $1 \mathrm{mg} . \mathrm{L}^{-1}$ et 0,22 mg. $\mathrm{L}^{-1}$ respectivement. Indépendamment de l'oxygène, le suivi des gaz dissous a mis en évidence des teneurs en $\mathrm{CO}_{2}$ très proches des $15-20 \mathrm{mg} . \mathrm{L}^{-1}$ considérées comme la limite à ne pas dépasser (Heinen et al 1996, Fivelstad et al 2003), malgré un rendement de $46 \%$ du système de dégazage assuré par l'injection d'air dans les filtres biologiques. Il conviendra de redimensionner l'injection d'air en cas de diminution du taux de renouvellement d'eau ou d'augmentation de charge dans les bassins. Le contrôle du $\mathrm{pH}$ par incorporation de bicarbonate de sodium à raison de $1 \mathrm{~kg}$ pour $5 \mathrm{~kg}$ d'aliment distribué a permis de tamponner suffisamment l'eau pour ne pas dégrader le rendement du filtre biologique. En ce qui concerne les éléments azotés, la robustesse du fonctionnement du filtre biologique a été surprenante quelles que soient les conditions d'élevage et de température. Les pratiques d'élevage couramment utilisées en $\mathrm{CO}$ intensif (mise à jeun avant tri, application de traitements antiparasitaires ou d'antibiothérapie), n'ont pas affecté son fonctionnement. Après une période de test sur le débit d'air injecté nous avons fixé celui-ci à $140 \mathrm{~m}^{3} \cdot \mathrm{h}^{-1}$. Depuis, ce filtre n'a nécessité aucun entretien. La restriction du débit de recirculation à 2,5 renouvellements par bac et par heure a permis de réduire la consommation énergétique des pompes sans dégrader la qualité de l'eau. Enfin, en ce qui concerne la modélisation des effluents du SR, les premiers résultats laissent apparaître que la méthode nutritionnelle peut être appliquée pour prédire la production de $\mathrm{N}-\mathrm{NH}_{4}{ }^{+}$et des MES, mais qu'elle nécessite des ajustements pour prédire la production de phosphore.

\section{4 / Performances de crois- sance}

Les performances zootechniques des truites arc-en-ciel (Oncorhynchus mykiss) élevées dans le CR ont été comparées à celles des truites arc-en-ciel élevées dans un système d'élevage classique en CO du 17/06/2011 au 25/04/2012 (P1) puis du 26/04/2012 au 04/12/2012 (P2). Les poissons qui ont servi aux expérimentations de croissance et de qualité de chair sont des truites arc-en-ciel triploïdes (98\% de triploïdes) d'une même cohorte, issue de femelles de souche Mirwart croisées avec des néo-males de souche Automnale (souches (C) INRA). Les poissons ont été acclimatés dans chaque système d'élevage 28 jours avant le début de cette étude (poids moyen d'introduction de $3,1 \mathrm{~g}$ pour les deux modalités d'élevage). A partir de cette cohorte, 3 lots ont été constitués dans chaque système d'élevage le 17/06/2011. Pour la seconde période $(26 / 04 / 2012)$ nous avons trié les lots de chaque système d'élevage en trois classes de taille (petite, moyenne et grosse) conformément aux pratiques professionnelles. Seuls les animaux de la classe de taille moyenne ont été conservés afin de reconstituer des lots homogènes dans les deux systèmes d'élevage. Le taux de renouvellement de l'eau dans les bassins du $\mathrm{CO}$ et du CR a été fixé à 2,5 par heure. L'augmentation de la biomasse de poissons a été estimée tous les mois dans les deux systèmes d'élevage par des pesées test $(10 \%$ des poissons prélevés 
Tableau 2. Performances zootechniques comparées pour les deux périodes et les deux systèmes d'élevage.

\begin{tabular}{|l|c|c|c|c|c|c|c|}
\hline & $\begin{array}{c}\text { Système } \\
\text { d'élevage }\end{array}$ & $\begin{array}{c}\text { Poids } \\
\text { initial (g) }\end{array}$ & $\begin{array}{c}\text { Poids } \\
\text { final (g) }\end{array}$ & IC & TCS (\%) & TGC (\%) & Survie (\%) \\
\hline \multirow{4}{*}{ Période 1 } & $\mathbf{C R}$ & 7,94 & $340 \pm 8,6$ & $0,91 \pm 0,02$ & $1,20 \pm 0,008$ & $2,79 \pm 0,072$ & $97,9 \pm 0,3$ \\
\cline { 2 - 8 } & $\mathbf{C O}$ & 6,93 & $303 \pm 7,9$ & $1,01 \pm 0,02$ & $1,19 \pm 0,009$ & $2,46 \pm 0,071$ & $96,9 \pm 1,8$ \\
\cline { 2 - 8 } & $P$ & $N S$ & 0,001 & 0,001 & 0,04 & 0,005 & $N S$ \\
\hline \multirow{3}{*}{ Période 2 } & $\mathbf{C R}$ & $340 \pm 1,14$ & $1335 \pm 24$ & $1,16 \pm 0,03$ & $0,76 \pm 0,01$ & $9,69 \pm 0,245$ & $96,4 \pm 0,2$ \\
\cline { 2 - 8 } & $\mathbf{C O}$ & $334 \pm 4,07$ & $1258 \pm 25$ & $1,35 \pm 0,1$ & $0,73 \pm 0,02$ & $9,36 \pm 0,285$ & $94,1 \pm 2,3$ \\
\cline { 2 - 8 } & $P$ & $N S$ & 0,006 & 0,018 & 0,01 & $N S$ & $N S$ \\
\hline
\end{tabular}

Toutes les valeurs sont des moyennes ( \pm écart type); P : Significatif $<0,05 ;$ NS : Non Significatif $; \mathrm{P}>0,05 ; n=3$ bacs par système d'élevage.

$\mathrm{CR}=$ Circuit Recirculé $; \mathrm{CO}=$ Circuit Ouvert $; \mathrm{IC}=$ quantité d'aliment distribuée sur une période donnée rapportée au gain de biomasse sur cette même période ; TCS $=100$ * $\left(\mathrm{Ln} \mathrm{pf}-\mathrm{Ln} \mathrm{pi)}{ }^{*}\right.$ nombre de jours ${ }^{-1}$; TGC $=\left(\mathrm{pf}^{1 / 3}-\mathrm{pi}^{1 / 3}\right) * 1000^{*}$ somme des degrés jours ${ }^{-1} ; \mathrm{pi}=$ poids initial $; \mathrm{pf}=$ poids final.

au hasard) ou par des pesées de l'ensemble des poissons du lot. Les poissons ont été mis à jeun 24 heures avant chaque pesée. Le taux de rationnement, la concentration en $\mathrm{O}_{2}$ en sortie de bac ainsi que la densité d'élevage (EOL: 0000043) dans les deux systèmes ont toujours été comparables. Cette dernière est passée de $15 \mathrm{~kg}$ de poisson par $\mathrm{m}^{3}$ en début d'expérimentation pour atteindre $75 \mathrm{~kg}$ en moyenne à partir du $120^{\mathrm{e}}$ jour de la première période. En seconde période la biomasse a été maintenue à une moyenne de $71 \mathrm{~kg} \cdot \mathrm{m}^{-3}$ grâce à des écrêtages successifs. Au début et en fin de chaque période ainsi que lors des écrêtages, une pesée totale et un dénombrement des animaux ont été réalisés.

Une synthèse des résultats est présentée dans le tableau 2. Pour les deux périodes, nous constatons que l'efficacité alimentaire a été améliorée par l'élevage en eau recirculée. Durant la première période, l'indice de consommation (IC, ATOL:0001580), c'est-à-dire la quantité d'aliment distribuée sur une période donnée rapportée au gain de biomasse sur cette même période, des animaux du CR a été de 0,91 et l'IC du CO de 1,01 . Durant la seconde période, l'IC du CR a été de 1,16 et l'IC du CO de 1,34. Pour chaque période, les performances de croissance des lots élevés dans le CR ont été supérieures à celles des lots élevés dans le CO. Le taux de croissance spécifique (TCS ${ }^{1}$, ATOL:0001662), a été significativement supérieur pour les lots élevés en CR. Enfin, le taux de survie est resté conforme aux standards de l'élevage et n'a pas été affecté par le système d'élevage.
La croissance des animaux élevés dans le système d'eau recirculée a été supérieure de $12 \%$ en première période et de $6 \%$ en seconde période à celle des animaux élevés en $\mathrm{CO}$. La température est un facteur essentiel pour la croissance des poissons et elle pourrait expliquer cette différence. Le coefficient thermique de croissance ("Thermal Growth Coefficient » ou TGC ${ }^{2}$, ATOL:0001661) permet en théorie de comparer la croissance des poissons élevés à des températures différentes. La somme de degrés jours sur la première période est de 3961 pour le CR et de 3720 sur le CO. En seconde période elle est respectivement de 3449 et de 3 179. Sur la première période le TGC est significativement plus élevé pour les animaux du CR que pour celui du CO. On ne peut donc pas imputer cette différence de croissance à la température d'élevage. Lors de la seconde période les TGC du CR et du CO n'ont pas été significativement différents. La différence de température peut donc expliquer cette croissance supérieure. Toutefois ce paramètre est critiquable lorsque les poissons subissent des températures supérieures à l'optimum thermique de l'espèce (Guillaume et al 1999a). Dans le cas de la truite arc-en-ciel on peut considérer que la température optimum est de $17^{\circ} \mathrm{C}$. Les animaux élevés dans le $\mathrm{CO}$ ont subi 27 jours de température supérieure à $17^{\circ} \mathrm{C}$ en $\mathrm{P} 1$ et 2 en $\mathrm{P} 2$ alors que dans le CR ils en ont subi 77 en P1 et 32 en P2. L'interprétation du TGC en première période reste donc délicate. Une température plus favorable notamment en période hivernale et printanière dans le CR pourrait compenser le nombre de jours où la température est défa- vorable en été. Un autre facteur à prendre en compte est la variation sur $24 \mathrm{~h}$ de la température. Dans le CO, la variation quotidienne de la température (EOL: $0000038)$ sur $24 \mathrm{~h}$ est très faible $\left(0,2^{\circ} \mathrm{C}\right)$ alors que dans le CR elle varie plus fortement notamment lors de journées ensoleillées $\left(+5^{\circ} \mathrm{C}\right)$. Cette augmentation de la température quelques heures après le dernier repas pourrait expliquer l'amélioration de l'indice de consommation car l'efficacité des enzymes digestives croit avec la température (Guillaume et al 1999b). Des expérimentations complémentaires avec des structures permettant de contrôler précisément la température seront nécessaires pour comprendre les mécanismes impliqués dans l'amélioration des performances de croissance des animaux élevés dans le SR. Au vu des résultats obtenus lors de cette expérimentation nous pouvons toutefois affirmer que le système d'élevage en CR ne dégrade pas mais au contraire améliore les performances de croissance obtenues dans un SO traditionnel.

\section{5 / Performances de repro- duction}

Afin d'évaluer les conséquences de ce système d'élevage sur la gestion d'un stock de reproducteurs, nous avons comparé les performances de reproduction de femelles issues d'un élevage en CR à celles issues d'un élevage en CO. Par ailleurs, la rémanence possible d'hormones stéroïdiennes sexuelles a été explorée par suivi de leurs concentrations dans les eaux des bassins. Deux bassins du CR ont été empoissonnés le 16 juin

\footnotetext{
1 TCS $=100 *($ Ln pf - Ln pi $) *$ nombre de jours ${ }^{-1}$ avec pi et pf le poids initial et final des poissons sur la période étudiée.

${ }^{2}$ Le TGC a été calculé selon l'équation : TGC $=\left(\mathrm{pf}^{1 / 3}-\mathrm{pi}^{1 / 3}\right) * 1000 *$ somme des degrés jours ${ }^{-1}$ avec pi et pf le poids initial et final des poissons sur la période étudiée.
} 
Tableau 3. Performances comparées des géniteurs dans les deux systèmes d'élevage.

\begin{tabular}{|c|c|c|c|c|}
\hline & $\begin{array}{c}\text { Poids des } \\
\text { femelles (g) }\end{array}$ & $\begin{array}{c}\text { Poids de 100 } \\
\text { ovules (g) }\end{array}$ & $\begin{array}{c}\text { Nb d'ovules } \\
\text { par femelle }\end{array}$ & $\begin{array}{c}\text { Nb d'ovules par } \\
\text { kg de poids vif }\end{array}$ \\
\hline CR & $1821 \pm 313,1$ & $4,86 \pm 0,59$ & $2734 \pm 912$ & $1453 \pm 539$ \\
\hline CO & $1244 \pm 186,1$ & $4,77 \pm 0,52$ & $2694 \pm 802$ & $1947 \pm 545$ \\
\hline$P$ & 0,000 & $N S$ & $N S$ & 0,005 \\
\hline
\end{tabular}

Toutes les valeurs sont des moyennes ( \pm écart type) ; P : Significatif $<0,05$; NS : Non Significatif : $P>0,05 ; n=21$ par système d'élevage.

Tableau 4. Performances de reproduction dans les deux systèmes d'élevage.

\begin{tabular}{|l|c|c|c|c|}
\hline & $\begin{array}{c}\text { Poids de } \\
100 \text { œufs } \\
(\mathrm{g})\end{array}$ & $\begin{array}{c}\text { Taux de } \\
\text { mortalité } \\
\text { à } 24 \mathrm{~h}(\%)\end{array}$ & $\begin{array}{c}\text { Taux de survie au } \\
\text { stade embryonné } \\
(\%)\end{array}$ & $\begin{array}{c}\text { Taux de } \\
\text { survie } \\
\text { à l'éclosion }\end{array}$ \\
\hline CR & $5,63 \pm 0,15$ & $1,1 \pm 0,4$ & $67,03 \pm 0,57$ & $46,35 \pm 1,10$ \\
\hline CO & $5,68 \pm 0,08$ & $1,2 \pm 0,2$ & $62,65 \pm 3,36$ & $44,68 \pm 3,41$ \\
\hline$P$ & $N S$ & $N S$ & $N S$ & $N S$ \\
\hline
\end{tabular}

Toutes les valeurs sont des moyennes ( \pm écart type) ; P : Significatif ; NS : Non Significatif ; $P<0,05 ; n=3$ par système d'élevage.

2010 avec 160 truites arc-en-ciel d'un poids moyen de $486 \mathrm{~g}$ (souche Automnale INRA). Elles ont été élevées dans le CR jusqu'à la reproduction $(02 / 11 / 2010)$. Le poids des femelles à la reproduction, le poids de 100 ovules, le nombre d'ovules par femelle (ATOL: 0000528) ont été comparés à ceux de truites issues du même lot élevées en CO (tableau 3). Le poids des femelles a été $30 \%$ plus important dans le SR que dans le système classique du fait d'une température plus favorable et d'une qualité physicochimique de l'eau plus stable. Le poids des ovules et le nombre d'ovules par femelle n'ont pas été différents.

La fécondation a été réalisée le 4 novembre 2010, deux jours après la détection de l'ovulation. Deux cent cinquante ovules de chaque femelle (mesurés par volumétrie) ont été repartis en trois pools. Toutes les femelles ont été fécondées par 21 mâles issus de chaque système d'élevage ( $\mathrm{CR}$ et $\mathrm{CO})$. Le taux de mortalité précoce, le taux de mortalité au stade embryonné, le taux de survie à l'éclosion ainsi que le poids des œufs (ATOL:0001880) ont été mesurés (tableau 4). Aucune différence significative n'est apparue dans les performances de reproduction que ce soit sur le taux de survie ou sur la taille des œufs. Le système d'élevage en CR n'a modifié en rien les performances de reproduction des géniteurs qui y ont terminé leur gamétogénèse.

Lors de cette expérimentation, des mesures de concentrations de stéroïdes sexuels ont été réalisées après extraction solide de 1,5 L d'eau et dosage radioimmunologique de l'extrait. Au début de la saison de reproduction (fin de vitellogenèse pour les femelles) des taux respectifs de 2,5 ng/L pour l'œstradiol et de $9,5 \mathrm{ng} / \mathrm{L}$ pour la testostérone ont été mesurés en sortie de bassin. Les abattements par le filtre biologique (différences entrée/sortie) restaient faibles avec des taux de $2 \%$ pour l'œstradiol et de $9 \%$ pour la testostérone. Un mois plus tard, alors que $50 \%$ des femelles ont donné des ovules, les concentrations en œstradiol étaient logiquement plus faibles $(1,6 \mathrm{ng} / \mathrm{L})$, l'œstradiol étant alors faiblement synthétisé par les femelles ovulantes, alors que les concentrations en testostérone se maintiennent $(12,2 \mathrm{ng} / \mathrm{L})$, ce qui est cohérent avec le pic de testostérone produit par l'ovaire juste avant l'ovulation. Les taux d'abattements par le filtre étaient alors nettement plus élevés que dans le mois qui précédait (53 et $43 \%$ respectivement pour l'œstradiol et la testostérone). Les concentrations de stéroïdes sexuels mesurées dans l'eau à différents points du circuit représentent un bilan ponctuel de plusieurs dynamiques : synthèse et sécrétion des stéroïdes par les génitrices, hydraulique du système, rétention par le filtre biologique, absorption/désorption par les matériaux utilisés pour la construction du circuit de recyclage. Par ailleurs, seules les concentrations de stéroïdes libres (fraction biologiquement active) ont été mesurées, mais les concentrations de stéroïdes conjugués devraient être plus élevées. Ces concentrations en stéroïdes libres restent faibles (plus de 1000 fois plus faibles que les taux circulants connus chez la truite arcen-ciel) et dans un contexte d'élevage, elles ne doivent pas poser problème; les femelles ayant d'ailleurs montré de bonnes performances de reproduction.

\section{6 / Gestion de la pression parasitaire}

Le contrôle de la pression parasitaire constitue un élément clé de la réussite économique d'un système d'élevage en eau recirculée car celle-ci peut être exacerbée par le recyclage, surtout quand l'eau qui alimente le circuit est une eau de surface potentiellement vectrice de parasites. Durant cette expérimentation, toutes les parasitoses ont été rigoureusement suivies et une attention particulière a été attachée au suivi et à la modélisation de la maladie des points blancs, ou parasitose à Ichthyophthirius multifiliis. Ce protozoaire infeste durant une phase de son cycle les épithéliums cutanés et branchiaux des poissons et peut provoquer des mortalités importantes. Il est difficile à contrôler en raison de son cycle de reproduction rapide et du fait de l'absence de sensibilité aux antiparasitaires des formes fixées sur les épithéliums. La mise en place de protocoles de surveillance et de traitement de l'infestation est donc très importante. Le formol est communément utilisé pour combattre cette parasitose, mais cette molécule est actuellement très controversée en raison de sa toxicité pour l'homme et l'environnement.

C'est pourquoi nous avons mis en place et essayé de mesurer l'efficacité d'un traitement alternatif à base d'acides peracétique et peroctanoïque qui a prouvé son efficacité en $\mathrm{CO}$ (Picon Camacho 2010). Les produits de dégradation de ces deux molécules $\left(\mathrm{O}_{2}, \mathrm{H}_{2} \mathrm{O}\right.$ et $\mathrm{CH}_{3}$ $\mathrm{COOH}$ ) sont sans danger pour l'homme et l'environnement. Ce présent travail avait pour objectif de suivre l'évolution de l'infestation parasitaire de truites élevées en système recirculé (CR) ou ouvert $(\mathrm{CO})$ en réponse à un protocole de traitement en utilisant un mélange d'acides peracétique et peroctanoïque et de vérifier que cet acide n'affectait pas la performance du filtre biologique du fait de ses propriétés antibactériennes et oxydantes. Le suivi parasitaire externe s'est déroulé sur une période de 264 jours (du 17/06/ 2011 au 16/02/2012) sur les animaux décrits au $\S 4$. Une fois par semaine, 4 poissons par bac ont été prélevés, soit 12 poissons par système d'élevage (CR ou $\mathrm{CO}$ ). Le prélèvement a été effectué de façon aléatoire, en utilisant du matériel préalablement désinfecté, et sur des poissons apparemment sains. Après constitution des deux pools de 12 individus, ces derniers ont été abattus par démédullation. L'échantillonnage a été réalisé par un frottis de mucus à l'aide d'une lamelle $\left(4 \mathrm{~cm}^{2}\right)$ le long de la ligne latérale du poisson depuis l'opercule jusqu'à la queue. Les lamelles ont ensuite été montées pour pouvoir être lues en utilisant une loupe binoculaire (grossissement 
25-50x) ou un microscope optique (grossissement 40x). Pour chacune de ces lamelles, une identification ainsi qu'un dénombrement des parasites ont été réalisés. Seuls les parasites identifiés comme Ichthyophthirius multifiliis ont fait l'objet d'un comptage exhaustif sur chaque lamelle. La présence éventuelle d'autres parasites (Gyrodactylus, Costia, Trichodine) a été relevée et notée sur les comptes rendus en terme de présence/absence et de niveau d'infestation jugé de façon qualitative (parasitose faible, moyenne ou forte). Le protocole préventif de traitement antiparasitaire suivi pour cette étude a consisté en un traitement bihebdomadaire par balnéation utilisant l'Incimaxx Aquatic ${ }^{\circledR}(\mathrm{ECOLAB}$, acide peracétique, $80 \mathrm{~g}^{\mathrm{kg}} \mathrm{kg}^{-1}$ et acide peroctanoïque $6 \mathrm{~g}^{\mathrm{kg}}{ }^{-1}$ ) à une concentration de $10 \mathrm{~mL} \cdot \mathrm{m}^{-3}$ soit $0,9 \mathrm{mg} . \mathrm{L}^{-1} \mathrm{~d}$ 'acide peracétique et $0,06 \mathrm{mg} \cdot \mathrm{L}^{-1} \mathrm{~d}$ 'acide peroctanoïque.

Sur les 648 poissons analysés au cours des 264 jours de l'essai, aucune parasitose par Ichthyophthirius multifiliis forte (de 31 à 75 parasites) ou très forte (> à 75 parasites) n'a été détectée et seul 1 poisson issu de chaque circuit a été atteint d'une parasitose moyenne (de 11 à 30 parasites). Par ailleurs, 51 poissons dans le CR et 22 poissons issus du CO ont été atteints d'une parasitose faible (de 1 à10 parasites). Les autres ne présentaient aucun parasite à l'examen. Aucune parasitose à monogène (Gyrodactylus) ou à trichodine, même faible, n'a été observée au cours des 264 jours de l'expérimentation. En revanche, un épisode de parasitose qualifiable de moyenne à forte par Costia Ichthybodo necator a été détecté le 30 juin 2011 simultanément dans les deux systèmes, entraînant le déclenchement d'un traitement par balnéation pendant 3 jours au formol (formaldéhyde à 23\%) à une dose de $250 \mathrm{~mL} \cdot \mathrm{m}^{-3}$.

Cette étude montre que le protocole de traitement préventif par utilisation d'un mélange d'acide peracétique et peroctanoïque par balnéation bihebdomadaire aux concentrations respectives de $0,9 \mathrm{mg} \cdot \mathrm{L}^{-1}$ d'acide peracétique et $0,06 \mathrm{mg} . \mathrm{L}^{-1} \mathrm{~d}$ 'acide peroctanoïque permet de contenir la pression parasitaire au sein d'un système en CR comme en circuit intensif ouvert classique. La comparaison entre le $\mathrm{CR}$ et le $\mathrm{CO}$ montre un portage légèrement plus important dans le cas du CR sans impact sur les performances des animaux ni sur la mortalité. Cependant, l'apparition au cours de l'expérimentation de deux épisodes de costiase dans les deux systèmes, indique aussi les limites de l'utilisation de ce mélange d'acides.

\section{7 / Qualité des produits}

La qualité des poissons des CR a été mesurée sur des truites arc-en-ciel tri- ploïdes aux stades portion $(\sim 350 \mathrm{~g})$ et grandes truites $(\mathrm{GT} \sim 1,3 \mathrm{~kg})$ sur deux cycles d'élevage consécutifs (de 2011 à 2012). Ces poissons ont été comparés à des congénères issus des mêmes pontes, et élevés en CO. La constitution des lots et les conditions d'élevage sont celles décrites dans le $\S 4$. Avant chaque abattage, les poissons ont été mis à jeun pendant $48 \mathrm{~h}$. Les critères de qualité mesurés ont été des paramètres morphométriques, l'état d'engraissement et les rendements (carcasse, filetage) pour les qualités technologiques. La qualité des filets a été évaluée par des mesures de $\mathrm{pH}$ musculaire, de couleur de la chair, de teneur en matières sèches et de résistance mécanique. Une synthèse des résultats est présentée dans le tableau 5.

\section{1 / Morphométrie}

La morphologie des poissons a été évaluée par mesure du poids vif des poissons (PV, ATOL:0000351), de leur longueur standard (L, ATOL:0001659) et calcul du coefficient de condition $\mathrm{K}=\mathrm{P} / \mathrm{L}^{3}$ (ATOL:0001653). Comme décrit précédemment, les poissons des CR sont plus gros que ceux des $\mathrm{CO}$ au stade portion, comme au stade GT. Pour l'abattage au stade GT de la deuxième cohorte, nous avons choisi de mesurer des poissons de PV comparable pour vérifier que les différences mesurées n'étaient pas dues uniquement à des différences de PV. Le coefficient de condition est apparu souvent différent entre les deux systèmes. Les poissons des CR ont un $\mathrm{K}$ supérieur aux poissons des $\mathrm{CO}$ dans la première cohorte au stade portion $(1,91 \pm 0,10$ vs $1,84 \pm 0,10)$ mais pas au stade GT. En revanche, l'année suivante, les poissons des CR ont un K plus faible que les poissons des $\mathrm{CO}$ aux deux stades mesurés $(1,63 \pm 0,10$ vs $1,73 \pm 0,13$ et $2,16 \pm 0,16$ vs $2,24 \pm 0,20)$. Ces résultats opposés ne permettent pas de conclure quant à un possible effet du CR sur la morphologie des poissons.

\section{2 / Adiposité}

L'adiposité musculaire des carcasses (ATOL:0001663) a été évaluée avec le fat-meter ${ }^{\circledR}$, aux stades portion et GT, respectivement. Sur la première cohorte, les poissons des $\mathrm{CR}$ apparaissent plus gras que ceux des CO aussi bien au stade portion que GT $(5,2 \pm 1,4$ vs $4,3 \pm$ 1,2 et $12,7 \pm 3,8$ vs $10,5 \pm 3,3$ en $\%$ de lipides). Ce résultat n'a pas été confirmé l'année suivante où nous n'observons pas de différence entre les poissons des $\mathrm{CR}$ et des $\mathrm{CO}$ sur ce paramètre. Les différences d'adiposité mesurées la première année peuvent être dues aux différences de poids entre les poissons des deux circuits puisque la différence n'est plus significative si l'analyse est faite avec le PV en covariable.

\section{3 / Rendements}

Le rendement en carcasse (ATOL: 0000548) des poissons des CR est significativement plus faible que celui des CO aux deux stades mesurés $(87,8 \pm 1,7$ vs $88,4 \pm 1,2$ et $83,6 \pm 2,5$ vs $85 \pm 2,1)$ pour la première cohorte et pour les GT de la deuxième année $(87,2 \pm 1,4 v s$ $88,2 \pm 1,4)$. Logiquement les rendements viscéro-somatiques (ATOL:0002259) sont donc plus élevés chez les poissons des CR dans ces mêmes expérimentations. Ce résultat est à rapprocher, au moins pour la première année, de la plus forte valeur de fat-meter mesurée pour les poissons des CR. Ceux-ci semblent avoir une adiposité globale plus élevée que ceux des $\mathrm{CO}$ avec plus de lipides au niveau musculaire ou sous-cutané (évalué par le fat-meter) et plus de tissu adipeux péri-viscéral qui augmente la proportion de viscères et dégrade le rendement en carcasse.

Aucun effet du CR n'a été trouvé sur le rendement en filet non paré non pelé (ATOL:0002305) pour la première année, ni au stade portion pour la deuxième année. En revanche, les poissons des CR de la deuxième expérimentation ont un rendement en filet non paré non pelé plus faible que ceux des $\mathrm{CO}$ au stade GT $(62,2 \pm 2,3$ vs $64 \pm 2,2)$. Ce résultat est en partie lié à la dégradation du rendement en carcasse chez les poissons des CR.

\section{4 / Qualité de la chair}

La qualité de la chair a été évaluée juste après l'abattage $(<2 \mathrm{~h})$ par une mesure de $\mathrm{pH}$ (avec une sonde à pénétration) (ATOL:0001684), de couleur (ATOL: 0001017 ) et de résistance mécanique du filet (ATOL:0001649) avec la peau. Les filets parés et pelés sont ensuite conservés sous vide à $4^{\circ} \mathrm{C}$ pendant $48 \mathrm{~h}$ avant une nouvelle mesure de $\mathrm{pH}$ (chair hachée diluée), de couleur (chromamètre Minolta), de teneur en matières sèches (ATOL:0000101) et de résistance mécanique (pénétrométrie avec un cylindre de $15 \mathrm{~mm}$ de diamètre et presse de Kramer). Un des filets est alors cuit au four à micro-ondes $\left(55^{\circ} \mathrm{C}\right.$ à cœur) et les mêmes mesures sont effectuées sur le filet cuit à 72 ou $96 \mathrm{~h}$ post-mortem.

\section{a) $\mathrm{pH}$}

Aucune différence de $\mathrm{pH}$ musculaire n'a été observée entre les poissons du CR et du CO.

\section{b) Couleur des filets}

L'effet du circuit d'eau sur la luminosité $\left(L^{*}\right)$ des filets est variable et ne permet pas de conclure quant à l'impact du système d'élevage sur ce paramètre. 
Tableau 5. Synthèse des effets de l'élevage en circuit recirculé sur la qualité des produits.

\begin{tabular}{|c|c|c|c|c|c|}
\hline & & \multicolumn{2}{|c|}{2011} & \multicolumn{2}{|c|}{2012} \\
\hline & & $\begin{array}{l}\text { Truite Portion } \\
\text { (environ } 350 \mathrm{~g} \text { ) }\end{array}$ & $\begin{array}{l}\text { Grande Truite } \\
\text { (environ } 1,3 \mathrm{~kg} \text { ) }\end{array}$ & $\begin{array}{l}\text { Truite Portion } \\
\text { (environ } 350 \mathrm{~g} \text { ) }\end{array}$ & $\begin{array}{l}\text { Grande Truite } \\
\text { (environ } 1,3 \mathrm{~kg} \text { ) }\end{array}$ \\
\hline \multicolumn{6}{|l|}{ Effectifs } \\
\hline \multirow{2}{*}{ Abattage } & poisson & 90 & 75 & 60 & 60 \\
\hline & filet & 90 & 75 & 30 & 30 \\
\hline \multicolumn{2}{|c|}{$48 \mathrm{~h}$ post mortem ( $\mathrm{pm}$ ) et filet cuit } & 30 & 24 & 15 & 15 \\
\hline \multicolumn{6}{|c|}{ Paramètres de qualité ; } \\
\hline \multicolumn{2}{|l|}{ Poids vif } & $\mathrm{CR}>\mathrm{CO}^{* * *}$ & $\mathrm{CR}>\mathrm{CO}^{* * *}$ & $\mathrm{CR}>\mathrm{CO}^{* * *}$ & $\mathrm{CR} \sim \mathrm{CO}^{(1)}$ \\
\hline \multicolumn{2}{|c|}{ Coefficient de condition $\mathrm{K}$} & $\mathrm{CR}>\mathrm{CO}^{* * *}$ & $\mathrm{CR} \sim \mathrm{CO}$ & $\mathrm{CR}<\mathrm{CO}^{* * *}$ & $\mathrm{CR}<\mathrm{CO}^{*}$ \\
\hline \multicolumn{2}{|l|}{ Fat-meter ${ }^{\circledR}$} & $\mathrm{CR}>\mathrm{CO}^{* * *}$ & $\mathrm{CR}>\mathrm{CO}^{* * *}$ & $\mathrm{CR} \sim \mathrm{CO}$ & $\mathrm{CR} \sim \mathrm{CO}$ \\
\hline \multicolumn{2}{|c|}{ Rendement en carcasse } & $\mathrm{CR}<\mathrm{CO}^{* *}$ & $\mathrm{CR}<\mathrm{CO}^{* * *}$ & $\mathrm{CR} \sim \mathrm{CO}$ & $\mathrm{CR}<\mathrm{CO}^{* * *}$ \\
\hline \multicolumn{2}{|c|}{ Rendement viscéro-somatique } & $\mathrm{CR}>\mathrm{CO}^{*}$ & $\mathrm{CR}>\mathrm{CO}^{* * *}$ & $\mathrm{CR} \sim \mathrm{CO}$ & $\mathrm{CR}>\mathrm{CO}^{* * *}$ \\
\hline \multicolumn{2}{|c|}{ Rendement en filet } & $\mathrm{CR} \sim \mathrm{CO}$ & $\mathrm{CR} \sim \mathrm{CO}$ & $\mathrm{CR} \sim \mathrm{CO}$ & $\mathrm{CR}<\mathrm{CO}^{* * *}$ \\
\hline \multirow{3}{*}{$\begin{array}{l}\text { Couleur } \\
\text { Abattage }\end{array}$} & $L^{*}$ & $\mathrm{CR}>\mathrm{CO}^{* *}$ & $\mathrm{CR} \sim \mathrm{CO}$ & $\mathrm{CR} \sim \mathrm{CO}$ & $\mathrm{CR} \sim \mathrm{CO}$ \\
\hline & $a^{*}$ & $\mathrm{CR} \geq \mathrm{CO}^{\mathrm{t}}$ & $\mathrm{CR}>\mathrm{CO}^{* *}$ & $\mathrm{CR} \sim \mathrm{CO}$ & $\mathrm{CR} \sim \mathrm{CO}$ \\
\hline & $b^{*}$ & $\mathrm{CR}>\mathrm{CO}^{* * *}$ & $\mathrm{CR} \sim \mathrm{CO}$ & $C R \geq C^{t}$ & $\mathrm{CR} \sim \mathrm{CO}$ \\
\hline \multirow{3}{*}{$\begin{array}{l}\text { Couleur } \\
48 \mathrm{~h} \text { post } \\
\text { mortem }\end{array}$} & $L^{*}$ & $\mathrm{CR} \geq \mathrm{CO}^{\mathrm{t}}$ & $\mathrm{CR} \sim \mathrm{CO}$ & $\mathrm{CR} \leq \mathrm{CO}^{\mathrm{t}}$ & $\mathrm{CR} \sim \mathrm{CO}$ \\
\hline & $a^{*}$ & $\mathrm{CR} \sim \mathrm{CO}$ & $\mathrm{CR}>\mathrm{CO}^{*}$ & $\mathrm{CR} \geq \mathrm{CO}^{\mathrm{t}}$ & $\mathrm{CR} \sim \mathrm{CO}$ \\
\hline & $b^{*}$ & $\mathrm{CR}>\mathrm{CO}^{* * *}$ & $\mathrm{CR} \sim \mathrm{CO}$ & $\mathrm{CR} \geq \mathrm{CO}^{\mathrm{t}}$ & $\mathrm{CR} \sim \mathrm{CO}$ \\
\hline \multirow{3}{*}{$\begin{array}{l}\text { Couleur } \\
\text { Filet cuit }\end{array}$} & $L^{*}$ & $\mathrm{CR} \sim \mathrm{CO}$ & $\mathrm{CR}<\mathrm{CO}^{*}$ & $\mathrm{CR} \sim \mathrm{CO}$ & $\mathrm{CR}<\mathrm{CO}^{*}$ \\
\hline & $a^{*}$ & $\mathrm{CR}>\mathrm{CO}^{* *}$ & $\mathrm{CR}>\mathrm{CO}^{\mathrm{t}}$ & $\mathrm{CR} \sim \mathrm{CO}$ & $\mathrm{CR} \sim \mathrm{CO}$ \\
\hline & $b^{*}$ & $\mathrm{CR} \sim \mathrm{CO}$ & $\mathrm{CR} \sim \mathrm{CO}$ & $\mathrm{CR} \sim \mathrm{CO}$ & $\mathrm{CR} \sim \mathrm{CO}$ \\
\hline \multirow{3}{*}{$\begin{array}{l}\text { Résistance } \\
\text { mécanique }\end{array}$} & Abattage & $\mathrm{CR}>\mathrm{CO}^{* * *}$ & $\mathrm{CR} \sim \mathrm{CO}$ & $\mathrm{CR}>\mathrm{CO}^{* *}$ & $\mathrm{CR} \sim \mathrm{CO}$ \\
\hline & $48 \mathrm{~h} p m$ & $\mathrm{CR} \geq \mathrm{CO}^{\mathrm{NS} \text { ou } * *(2)}$ & $\begin{array}{c}\mathrm{CR} \geq \mathrm{CO}^{\mathrm{NS} \text { ou } *(2)} \text { median } \\
\mathrm{CR}>\mathrm{CO}^{* *} \text { caudale }\end{array}$ & $\mathrm{CR}>\mathrm{CO}^{* *}$ ou $* * *(2)$ & $\mathrm{CR} \sim \mathrm{CO}$ \\
\hline & Filet cuit & $\mathrm{CR}<\mathrm{CO}^{*}$ & $\begin{array}{l}\mathrm{CR}>\mathrm{CO}^{*} \text { median } \\
\mathrm{CR}<\mathrm{CO}^{*} \text { caudale }\end{array}$ & $\mathrm{CR} \sim \mathrm{CO}$ & $\mathrm{CR} \sim \mathrm{CO}$ \\
\hline \multirow{3}{*}{$\mathrm{pH}$ musculaire } & Abattage & $\mathrm{CR} \sim \mathrm{CO}$ & $\mathrm{CR} \sim \mathrm{CO}$ & $\mathrm{CR} \sim \mathrm{CO}$ & $\mathrm{CR} \sim \mathrm{CO}$ \\
\hline & 48h $p m$ & $\mathrm{CR} \sim \mathrm{CO}$ & $\mathrm{CR} \sim \mathrm{CO}$ & $\mathrm{CR} \sim \mathrm{CO}$ & $\mathrm{CR} \sim \mathrm{CO}$ \\
\hline & Filet cuit & $\mathrm{CR} \sim \mathrm{CO}$ & $\mathrm{CR} \sim \mathrm{CO}$ & $\mathrm{CR} \sim \mathrm{CO}$ & $\mathrm{CR} \sim \mathrm{CO}$ \\
\hline \multirow{2}{*}{$\begin{array}{l}\text { Matières } \\
\text { sèches }\end{array}$} & Filet cru & $\mathrm{CR} \sim \mathrm{CO}$ & $\mathrm{CR}>\mathrm{CO}^{* *}$ & $\mathrm{CR} \sim \mathrm{CO}$ & $\mathrm{CR} \sim \mathrm{CO}$ \\
\hline & Filet cuit & $\mathrm{CR} \sim \mathrm{CO}$ & $\mathrm{CR} \geq \mathrm{CO}^{\mathrm{t}}$ & $\mathrm{CR}<\mathrm{CO}^{*}$ & $\mathrm{CR} \sim \mathrm{CO}$ \\
\hline
\end{tabular}

\footnotetext{
${ }^{\mathrm{t}}$ : tendance $0,05 \leq \mathrm{P}<0,10 ;{ }^{*}: \mathrm{P}<0,05 ;{ }^{* *}: \mathrm{P}<0,01 ;{ }^{* *}: \mathrm{P}<0,001$.

(1) Les poissons prélevés pour les mesures de qualité ont été volontairement choisis de taille similaire entre les deux systèmes.

${ }^{(2)}$ Selon la méthode de mesure.
}

Pour ce qui est des composantes rouge $\left(a^{*}\right)$ et jaune $\left(b^{*}\right)$, l'effet n'est pas systématique selon l'expérimentation, le stade, ou le moment de la mesure. Néanmoins quand un effet est mesuré, il l'est toujours dans le sens d'un filet plus coloré (plus rouge ou plus jaune) chez les poissons du CR que chez ceux du CO. Ce résultat peut être rapproché de la tendance à une plus forte adiposité musculaire des poissons du CR puisque les pigments caroténoïdes, responsables de la pigmentation, sont liposolubles et donc transportés par les lipides. Néanmoins, cet effet n'a pas été observé pour les GT de la deuxième cohorte et n'est donc pas systématique.

\section{c) Résistance mécanique de la chair}

La résistance mécanique des filets, qui est un indicateur de fermeté, montre qu'un effet du système d'élevage peut être observé sur ce paramètre mais pas systématiquement. Quand un effet est observé, il est toujours dans le sens d'une plus grande fermeté des filets crus des poissons des CR. Pour ce qui est des filets cuits, les résultats sont plus contradictoires.

d) Teneur en Matières Sèches (MS)

La teneur en MS permet d'évaluer la teneur en lipides musculaires, inversement corrélée avec la teneur en MS, 
plus facilement qu'avec un dosage des lipides. Pour la première cohorte, des teneurs plus élevées en matières sèches ont été mesurées pour les filets des GT élevées en CR $(33,5 \pm 3,2$ vs $31,1 \pm 2,0)$. Ce résultat est cohérent avec les plus fortes valeurs de fat-meter obtenues chez ces animaux. De façon plus étonnante, une plus faible teneur en MS des filets cuits des truites portion élevées en CR a été mesurée sur la deuxième cohorte $(27,4 \pm 1,2$ vs $28,2 \pm 0,5)$.

\section{e) Analyse sensorielle}

Des filets des GT de la deuxième cohorte élevée en $\mathrm{CR}$ et $\mathrm{CO}$ ont été soumis à un jury non entrainé d'analyse sensorielle pour un test de profil permettant de noter des critères d'aspect (ATOL:0000064), de flaveur (ATOL: 0001002) et de texture (ATOL:0001054). Cette analyse n'a révélé aucune différence significative entres les filets des $\mathrm{CR}$ et des $\mathrm{CO}$, quelque soit le paramètre évalué. En particulier aucune " off-flavor », odeur de vase très défavorable qui peut apparaître dans des conditions d'élevage en CR, n'a été détectée par le jury.

\section{5 / Discussion}

Très peu d'études rapportent des mesures de qualité sur des poissons élevés en $\mathrm{CR}$ et aucune ne les compare à des congénères élevés en $\mathrm{CO}$. Les travaux portant sur des poissons élevés en $\mathrm{CR}$ portent principalement sur les effets comparés de différentes qualités d'eau ou de pratiques d'élevage (densité, nature de l'aliment, rationnement) sur la croissance et la qualité des poissons (David 2006, Roque d'Orbcastel et al 2009b, Davidson et al 2013). Le principal paramètre de qualité ciblé est l'apparition d' " offflavor " et la possibilité de les éliminer par différents traitements (Burr et al 2012, Auffret et al 2013). Quoi qu'il en soit, la comparaison de poissons issus de deux systèmes d'élevage intègre les effets de nombreux facteurs environnementaux pouvant influer sur la qualité du produit. Dans cette étude, nous avons essayé de limiter ces facteurs aux seules différences liées au circuit d'eau, en comparant, entre $\mathrm{CR}$ et $\mathrm{CO}$, des poissons issus d'une même cohorte et avec les mêmes pratiques alimentaires (rationnement, mode distribution de l'aliment). Les principales différences entre les deux systèmes étaient donc la qualité de l'eau et le profil thermique. D'après les résultats zootechniques (cf. § 4), la plus forte croissance des poissons des CR peut expliquer des différences de qualité du produit. La plus forte adiposité des truites élevées en CR affecte les rendements (carcasse et filet) et peut modifier la couleur de la chair (Bugeon et al 2010). Une croissance globale plus rapide peut avoir des conséquences sur l'organisation du tissu musculaire (augmentation du nombre et de la taille des fibres musculaires) et engendrer des différences de texture (Lefèvre et al 2008). Parmi l'ensemble des paramètres qui diffèrent entre les deux systèmes, on peut penser que les différences de profils thermiques de l'eau sont primordiales. Pour évaluer un effet possible de la différence de qualité de l'eau, il faudrait pouvoir contrôler la température dans le CR pour appliquer un profil identique à celui du CO. Les différences de qualité des produits observées entre les deux circuits restent faibles et non systématiques. Par exemple, le fait que le $\mathrm{pH}$ musculaire ne soit jamais affecté suggère que ni le métabolisme musculaire des poissons ni leurs réserves énergétiques musculaires ne sont affectés par le système d'élevage.

Ce travail montre que les effets du système d'élevage sur la qualité de la chair sont globalement peu importants et l'élevage en CR peut donc être considéré comme un moyen de produire des truites de qualité similaire à celles élevées en $\mathrm{CO}$.

\section{Conclusions et perspectives}

Le travail que nous avons réalisé a permis de valider la faisabilité technique d'un système d'élevage utilisant le principe de la recirculation de l'eau pour une production de salmonidés. Les résultats montrent que le pilote mis en place à la PEIMA a atteint voire dépassé l'objectif de réduction d'un facteur 10 de la consommation d'eau neuve. La quantité d'eau nécessaire n'a été que de 7,2 $\pm 0,9 \mathrm{~m}^{3}$ par kilo de poisson produit dans le CR contre $102 \pm 5 \mathrm{~m}^{3}$ d'eau pour le CO. Nous avons constaté que les principaux indicateurs zootechniques étaient significativement améliorés dans le CR et les résultats en termes de qualité de la chair ne laissent apparaitre que peu de différence entre les deux systèmes d'élevage (Lefèvre et al 2012). Enfin, aucune pathologie bactérienne ou parasitaire majeure n'a été mise en évidence par le vétérinaire aquacole qui a suivi ce projet.

Ces résultats ont été obtenus à une densité moyenne de poissons de $75 \mathrm{~kg} / \mathrm{m}^{3}$. Cette élévation de la production par unité de surface alliée à l'amélioration des résultats zootechniques a démontré que nous pouvions optimiser la capacité de production d'un site en ajoutant une boucle de traitement de l'eau et en utilisant des techniques et des équipements déjà présents dans le système intensif classique. L'utilisation de techniques éprouvées de filtration et d'oxygénation de l'eau, alliées à la robustesse du fonctionnement du filtre biologique à lit fluidisé, n'a ni complexifié ni alourdi la charge de travail pour les éleveurs. Nous noterons toutefois que ce système d'éle- vage nécessite la mise en pratique de techniques zootechniques de précision (modélisation de la croissance, gestion de l'alimentation, suivi de la qualité d'eau, suivi de la pathologie). Un transfert des connaissances issues de la recherche vers les professionnels et l'enseignement sera nécessaire afin que les éleveurs puissent s'approprier et utiliser ces techniques d'élevage.

L'utilisation de CR pour le grossissement de truites arc en ciel passera aussi par une amélioration de l'efficacité du système. Il sera nécessaire de perfectionner la boucle du traitement de l'eau et de valoriser les effluents et les nutriments produits par le SR. D'une manière globale, la consommation énergétique d'un CR est de 1,4 à 2,8 fois plus importante que celle d'un CO (Roque d'Orbcastel et al 2009b). La réduction de la consommation d'énergie est possible en trouvant un compromis entre une conception optimale d'un circuit pour la circulation de l'eau, la gestion des gaz $\left(\mathrm{O}_{2}, \mathrm{CO}_{2}\right)$ et le fonctionnement du filtre biologique. Des recherches récentes portant sur des systèmes d'air lift à dépression et à basse consommation d'énergie (Barut 2011) ont démontré qu'il était possible d'améliorer le traitement des gaz (élimination du $\mathrm{CO}_{2}$ ) et d'éliminer les MES les plus fines. Une autre voie concernant la formulation d'aliment sera testée. L'incorporation de liants tels que le "guar» dans l'aliment augmente la stabilité des fèces et permet de capter plus facilement les MES tout en limitant la solubilisation du phosphore et de l'azote qu'elles contiennent (Binker et al 2005). Ces actions sur les MES devraient orienter le développement de la flore bactérienne autotrophe au détriment de la flore hétérotrophe du filtre biologique et ainsi augmenter son efficacité (Michaud 2007).

De nouvelles voies de prophylaxie permettant de contenir la pression parasitaire ou de pathogènes seront explorées. $\mathrm{Du}$ fait de la forte réduction des quantités d'eau nécessaires, le traitement de «l'eau neuve » entrant dans le système devient envisageable techniquement et économiquement. Des systèmes de traitement aux ultra-violets, à l'ozone $\left(\mathrm{O}_{3}\right)$ ou encore de filtration mécanique fine ( $1 \mu \mathrm{m}$ ou inférieur) seront testés. Ces techniques, associées à l'utilisation d'animaux sains permettraient de limiter le recours à l'utilisation de biocides destinés à prévenir les attaques parasitaires ou bactériennes.

Enfin, nous devons aussi nous attacher à la valorisation des effluents issus de ce système. La mise en place et l'évaluation d'outils de traitement et de valorisation spécifiques aux SR est actuellement en cours à la PEIMA. Nous explorons notamment des techniques de 
récupération rapide et de concentration des matières solides en suspension permettant de les valoriser par co-compostage. Par ailleurs, si nous pouvons actuellement abattre une grande partie de l'ammoniaque produit et ainsi respecter les normes de rejets pour les piscicultures, il nous faudra inclure dans la boucle de traitement des effluents un système de dénitratation. Les deux pistes explorées sont : i) une voie bactérienne anaérobie avec utilisation d'une source de carbone interne et ii) l'utilisation d'un modèle d'aquaculture intégrée multi-trophique, l'aquaponie. Ce système vise à valoriser les rejets de nutriments solubles par des cultures hydroponiques à valeur ajoutée (légumes, fruits, fleurs).

Ces améliorations techniques, associées à l'appropriation par les éleveurs de pratiques zootechniques de précision, devraient à terme permettre à la filière de pouvoir évoluer vers un mode de production plus durable, économiquement viable, tout en continuant à fournir des produits de qualité aux consommateurs.

\section{Remerciements}

Les auteurs remercient le personnel de la PEIMA qui s'est fortement impliqué dans le suivi des expérimentations réalisées dans ce pilote et plus particulièrement Vincent Gayet qui a participé à sa conception et à sa réalisation. Ce projet a été labellisé par le pôle de compétitivité Mer Bretagne et financé par le département PHASE (crédits incitatifs Top Down), la Région Bretagne, l'Europe (fonds FEDER) et le Conseil général du Finistère.

\section{Références}

Auffret M., Yergeau E., Pilote A., Proulx E., Proulx D., Greer C.W., Vandenberg G., Villemur R., 2013. Impact of water quality on the bacterial populations and off-flavours in recirculating aquaculture systems. FEMS Microbiol. Ecol., 84, 235-247.

Barrut B., 2011. Etude et optimisation du fonctionnement d'une colonne airlift à dépression Application à l'aquaculture. Thèse de l'Université Montpellier II, France, 154p. http://archimer.ifremer.fr/doc/00050/16091/13979.pdf

Blancheton J.P., Bosc P., Hussenot J., Roque d'Orbcastel E., Romain D., 2009. Tendances pour la pisciculture européenne de demain : cages au large, systèmes en eau recirculée et systèmes intégrés. Cah. Agricult., 18, 227-234.

Brinker A., Koppe W., Rösch R., 2005. Optimised effluent treatment by stabilised trout faeces. Aquaculture 249, 125-144.

Bugeon J., Lefèvre F., Cardinal M., Uyanik A., Davenel A., Haffray P., 2010. Flesh quality in large rainbow trout with high or low fillet yield. J. Muscle Foods, 21, 702-721.

Burr G.S., Wolters W.R., Schrader K.K., Summerfelt S.T., 2012. Impact of depuration of earthy-musty off-flavors on fillet quality of Atlantic salmon, Salmo salar, cultured in a recirculating aquaculture system. Aquacult. Eng., 50, 28-36.

David J.A., 2006. Water quality and accelerated winter growth of European catfish using an enclosed recirculating system. Water Environ. J., 20, 233-239.

Davidson J., Good C., Barrows F.T., Welsh C., Kenney P.B., Summerfelt S.T., 2013. Comparing the effects of feeding a grain- or a fish mealbased diet on water quality, waste production, and rainbow trout Oncorhynchus mykiss performance within low exchange water recirculating aquaculture systems. Aquacult. Eng., $52,45-57$.

DCE, 2000. Directive 2000/60/CE du Parlement européen et du Conseil du 23 octobre 2000 établissant un cadre pour une politique communautaire dans le domaine de l'eau. http://eurlex.europa.eu/LexUriServ/LexUriServ.do?uri= OJ:L:2000:327:0001:0072:fr:PDF

FAO, 2011. La situation mondiale des pêches et de l'aquaculture 2011. Département des pêches FAO. http://www.fao.org/docrep/016/i2727f/ i2727f01.pdf

Fauré A., Labbé L., 1999. Pratique de l'alimentation des poissons. In : Nutrition et alimentation des poissons et crustacés. Éditions INRAIFREMER, 489, 412-426.
Fivelstad S., Olsen A., Åsgård T., Bæverfjord G., Rasmussen T., Vindheim T., Stefansson S.O., 2003. Long-term sub-lethal effects of carbon dioxide on Atlantic salmon smolts: ion regulation, haematology, element composition, nephrocalcinosis and growth parameters. Aquaculture. 215, 301-319.

France Agrimer, 2011. Commerce extérieur des produits de la pêche et de l'aquaculture. Statistiques 2011. http://www.franceagrimer.fr/ content/download/16518/126305/file/STAMER-CONSO-2011.pdf

Golik W., Dameron O., Bugeon J., Fatet A., Hue I., Hurtaud C., Reichstadt M., MeunierSalaün M.C., Vernet J., Joret L., Papazian F., Nédellec C., Le Bail P.Y., 2012. ATOL: the multi-species livestock trait ontology. $6^{\text {th }}$ Int. Conf. Metadata Semantic Res. (MTSR'12), Cadiz, Spain, 2012/11/28-30.

Guillaume J., Kaushik S., Bergot P., Metailler R., 1999a. Terminologie et rappels méthodologiques. In : Nutrition et alimentation des poissons et crustacés. Editions INRA-IFREMER, $489,32-40$

Guillaume J., Kaushik S., Bergot P., Metailler R., 1999b. Bases de la nutrition des animaux aquatiques : physiologie digestive et digestibilité des nutriments. In : Nutrition et alimentation des poissons et crustacés. Éditions INRAIFREMER, 489, 71-75.

Heinen J.M., Hankins J.A., 1996. Water quality and waste production in a recirculating trout culture system with feeding of a higher-energy or a lower-energy diet. Aquacult. Res., 27, 699710 .

Kristensen T., Åtland Å., Rosten T., Urke H.A., Rosseland B.O., 2009. Important influent-water quality parameters at freshwater production systems in two salmon producing countries. Aquacult. Eng., 41, 53-59.

Lefèbvre F., Bugeon J., Auperin B., Aubin J., 2008. Rearing oxygen level and slaughter stress effects on rainbow trout flesh quality. Aquaculture, 284, 81-89.

Lefèvre F., Colson V., Bugeon J., Gaume M., Valotaire C., Labbe L., 2012. Poster. In : $3^{\text {emes }}$ Journ. Rech. Filière Piscicole, 25.

LEMA., 2006. Loi n²006-1772 du 30 decembre 2006 portant transposition de la directive 2000/60/CE du Parlement européen et du Conseil du 23 octobre 2000 établissant un cadre pour une politique communautaire dans le domaine de l'eau. http://www.legifrance.gouv.fr /affichTexte.do?cidTexte=JORFTEXT $\underline{000000649171 \& \text { dateTexte }=\& \text { categorieLien }=\mathrm{id}}$
Martins C.I.M., Ending E.H., Verdegem M.C.J., Heinsbroek L.T.N., Schneider O., Blancheton J.P., Roque d'Orbcastel E., Verreth J.A.J., 2010. New developments in recirculating aquaculture systems in Europe: A perspective on environmental sustainability. Aquacult. Engin., 43, 83-93.

Michaud L., 2007. Microbial communities of recirculating aquaculture facilities: interaction between heterotrophic and autotrophic bacteria and the system itself. Thèse de doctorat. Université Montpellier II, 142p. http://archimer.ifremer.fr/doc/2007/these-2682.pdf

Papatryphon E., Petit J., Hayo V., Kaushik S.J., Claver K., 2005. Nutrient balance modelling as a tool for environmental management in aquaculture: The case of trout farming in France. J. Environ. Management, 35, 161-174.

Roque d'Orbcastel E., 2008. Optimisation de deux systèmes de production piscicole : biotransformation des nutriments et gestion des rejets. Thèse de doctorat. Toulouse : Institut National Polytechnique, 144p. http://ethesis inp-toulouse.fr/archive $/ 00000632 / 01 /$ roque d orbcastel.pdf

Roque d'Orbcastel E., Person-le Ruyet J., Le bayon N., Blancheton J.P., 2009a. Comparative growth and welfare in rainbow trout reared in recirculating and flow through rearing systems. Aquacult. Eng. 40, 79-86.

Roque d'Orbcastel E., Blancheton J.P., Aubin J., 2009b. Towards environmentally sustainable aquaculture: Comparison between two trout farming systems using Life Cycle Assessment. Aquacult. Eng., 40, 113-119.

Schneider O., Blancheton J.P., Varadi L., Eding E.H., Verreth J.A.J., 2006. Cost price and production strategies in European recirculation systems. Linking Tradition \& Technology Highest Quality for the Consumer. WAS. Firenze, Italy.

Picón Camacho SM., 2010. Developing strategies for the control of ichthyophthirius multifiliis. Thesis submitted for the degree of doctor of philosophy institute of aquaculture university of Stirling. 258p. https://dspace.stir.ac.uk/ bitstream/1893/3032/1/Sara\%20Picon\%20 Camacho\%20PhD\%20thesis.pdf

Tanguy H., 2008. Rapport final de la mission sur le développement de l'aquaculture. http:// agriculture.gouv.fr/IMG/pdf/Rapport H Tanguy . $\mathrm{pdf}$

Van Duijn A.P., Schneider O., Poelman M., van der Veen H., Beukers R., 2010. Visteelt in Nederland: analyse en aanzet tot actie. Den Haag, LEI, Wageningen, 56p. 


\title{
Résumé
}

La faisabilité technique d'un système d'élevage de salmonidés en eau recirculée et ses performances ont été comparées, sur 3 ans, à

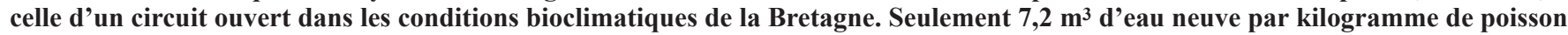
produit ont été nécessaires au lieu de $100 \mathrm{~m}^{3}$ en circuit ouvert. Le dimensionnement des composants du pilote a permis cette forte réduction sans dégradation de la qualité de l'eau d'élevage $\left(\mathrm{N}-\mathrm{NH}_{4}^{+}: 0,66 \pm 0,13 \mathrm{mg} . \mathrm{L}^{-1} ; \mathrm{N}-\mathrm{NO}_{2}^{-}: 0,12 \mathrm{mg} . \mathrm{L}^{-1} \pm 0,03\right)$. Les principaux indicateurs zootechniques ont été significativement améliorés (de 10 à $15 \%$ de croissance pondérale supplémentaire avec un indice de consommation diminué de près de $10 \%$ ) sans impact sur la mortalité. La reproduction n'a pas été affectée par ce système d'élevage (taux de survie des œufs embryonnés : $67,03 \pm 0,57 \%$ vs $62,65 \pm 3,36 \%$ ) et le parasitisme a pu être contrôlé. La qualité de la chair, et notamment la flaveur du filet, n'a pas été altérée. Enfin, l'impact de ce système sur le rendement au filetage et la qualité physicochimique du filet (texture, couleur, teneur en lipides) reste faible. Malgré un impact environnemental réduit, ce système de production est encore peu développé en Europe. La combinaison judicieuse d'améliorations techniques dans la boucle de recirculation et de traitement de l'eau, visant à améliorer l'efficacité du système, pourrait aider la filière piscicole à évoluer vers un mode de production moins dépendant des ressources naturelles, tout en continuant à fournir des produits de qualité au consommateur.

\begin{abstract}
Rainbow trout farming in Recirculating Aquaculture System (RAS): An innovative and environmental friendly system.

The objective of this project was to test the feasibility of a recirculated aquaculture system in the bioclimatic conditions of the PEIMA. During the 3 year experiment, only $7.2 \mathrm{~m}^{3}$ of make-up water per kilogram of fish produced has been used instead of the 100 required for an open output circuit. The design of the system components allowed this sharp reduction without degrading water quality $\left(\mathrm{N}-\mathrm{NH}_{4}^{+}: 0.66 \pm 0.13 \mathrm{mg} . \mathrm{L}^{-1} ; \mathrm{N}-\mathrm{NO}_{2}^{-}: 0.12 \mathrm{mg} . \mathrm{L}^{-1} \pm 0.03\right)$. The main indicators of animal performances were significantly improved (10 to 15\% more weight gain and feed consumption ratio decreased by nearly $10 \%$ ) and no impact on survival rate was observed. Reproduction was not affected by the rearing system (eggs survival rate: $67.03 \pm 0.57 \%$ vs $62.65 \pm 3.36 \%$ ) and parasitism could be controlled. Flesh quality such as flavor has not been altered. Fillet and physicochemical quality of fillet (colour, texture, fat content) are only slightly affected by the rearing system. Despite its environmentally friendly characteristics its contribution to the European production is still small compared to cages, flow-through systems or ponds. The judicious combination of technical improvements in the recirculation loop and water treatment could improve the efficiency of the system in order to help the aquaculture industry to be less dependent on natural resources, while continuing to provide quality products to the consumer.
\end{abstract}

LABBÉ L., LEFÈVRE F., BUGEON J., FOSTIER A., JAMIN M., GAUMÉ M., 2014. Conception d'un système innovant de production de truites en eau recirculée. In : Numéro spécial, Quelles innovations pour quels systèmes d'élevage ? Ingrand S., Baumont R., (Eds). INRA Prod. Anim., 27, 135-146. 